\title{
Author Correction: Donor lymphocyte infusions for B-cell malignancies relapse after T-cell replete allogeneic hematopoietic cell transplantation
}

\author{
Guillermo Ortí ${ }^{1}$ Irene García-Cadenas ${ }^{2}$ - Lucia López-Corral ${ }^{3}$ - Ariadna Pérez ${ }^{4} \cdot$ Maria Jose Jimenez $^{5}$. \\ Isabel Sánchez-Ortega ${ }^{6} \cdot$ Laura Alonso $^{7} \cdot$ Luisa Sisinni $^{8} \cdot$ Laura Fox $^{1} \cdot$ Guillermo Villacampa $^{9} \cdot$ Isabel Badell $^{8}$. \\ Cristina Diaz de Heredia ${ }^{7}$. Rocio Parody $\mathbb{1 0}^{6} \cdot$ Christelle Ferrà (it) $^{5} \cdot$ Carlos Solano $^{4} \cdot$ Dolores Caballero $^{3}$. \\ Rodrigo Martino ${ }^{2} \cdot$ Sergi Querol ${ }^{10} \cdot$ David Valcárcel $^{1}$
}

Published online: 7 February 2019

(c) Springer Nature Limited 2019

Correction to: Bone Marrow Transplantation (2018); https://doi.org/10.1038/s41409-018-0422-z; published online 13 December 2018
In the original version of this article, author 'Lucia López-Corral' was incorrectly listed as 'Lucia López'. This has now been corrected in both the PDF and HTML versions of the article to 'Lucia López-Corral'.
Guillermo Ortí

gorti@vhebron.net

1 Hematology Department, Hospital Universitari Vall d’Hebron, Departament de Medicina, Universitat Autonoma de Barcelona, Barcelona, Spain

2 Hematology Department, Hospital de la Santa Creu i Sant Pau, José Carreras Leukemia Research Institute and IIB Sant Pau, Universitat Autònoma de Barcelona, Barcelona, Spain

3 Hematology Department, Hospital Universitario de SalamancaIBSAL, Salamanca, Spain

4 Hematology Department, Hospital Clínico de Valencia, Valencia, Spain

5 Hematology Department, Institut Català d'Oncologia-Hospital
Universitari Germans Trias i Pujol, Jose Carreras Research Institute, Barcelona, Spain

6 Hematology Department, Institut Català d'Oncologia, Hospital Duran i Reynals, Barcelona, Spain

7 Paediatric Oncology and Hematology Department, Hospital Universitario Vall d'Hebron, Universitat Autonoma de Barcelona, Barcelona, Spain

8 Paediatric Oncology and Haematology Department, Hospital de la Santa Creu i Sant Pau, Universitat Autònoma de Barcelona, Barcelona, Spain

9 Oncology Data Science (ODysSey) Group, Vall d'Hebron Institute of Oncology, Barcelona, Spain

10 Banc de Sang i Teixits de Barcelona, Barcelona, Spain 